\title{
Editorial
}

\section{Bacille Calmette-Guérin Supply Disruption Emphasizes the Importance of Stewardship}

\author{
Alejandro Abello ${ }^{1}$ Patrick A. Kenney ${ }^{1}$ \\ ${ }^{1}$ Department of Urology, Yale School of Medicine/Yale-New Haven \\ Hospital, New Haven, CT, United States
}

Urol Colomb 2019;28:191-192.

Since the original trial published by Morales et al in 1976, intravesical Bacille Calmette-Guérin (BCG) has been a centerpiece in non-muscle invasive bladder cancer (NMIBC) treatment, with a rigorous induction and maintenance regimen shown to decrease cancer recurrence and progression. ${ }^{1,2}$ More than $50 \%$ of the 81,000 new cases of bladder cancer that are diagnosed each year in the United States (US) (550,000 in the world) are NMIBCs, and it is clear that effective and timely treatments like BCG are essential and must be readily available.,

A combination of increased bladder cancer incidence, early diagnosis of bladder cancer, drug properties, and manufacturing setbacks have contributed to recurrent BCG shortages. ${ }^{5}$ In addition, shortages are influenced by the unusual nature of BCG, a live microorganism that requires a sterile environment and between 1 to 3 months for a batch to grow. ${ }^{6}$ Furthermore, the quantity required for BCG instillation is much higher than for tuberculosis vaccination, the other primary use of BCG. It has been estimated that the quantity required for one induction course would be enough to vaccinate between 10,000 and 100,000 people. ${ }^{7}$ The shortages impact patient care. Khanna et al demonstrated a decrease of BCG utilization ranging from $0.62 \%$ increase per year in the period from 2004 to 2012 to $0.29 \%$ increase per year in the period between 2013 and 2015, highlighting the negative impact of supply disruptions. ${ }^{5}$

Other factors, such as production challenges and restricted supply, have contributed to BCG disruptions. Only two BCG manufacturers had been approved for use in the US; Sanofi (Connaught strain) and Merck (TICE strain). In 2011, Sanofi's Connaught strain production was suspended by the Food and Drug Administration (FDA) after mold contamination was detected in the production plant. Sanofi stopped manufacturing BCG for North America in 2017, further endangering the supply of the drug at a time of rising demand and leaving Merck as the sole supplier of BCG in the
US. Merck has also had production challenges. In 2014, a strain contamination halted the production of Merck's TICE, thus causing shortages in North and South America. Recently, Merck announced renewed supply constraints during the first quarter of $2019 .^{8}$

In this new era of BCG shortage, proper disease stratification, correct medication selection, and patient preparation with optimal transurethral resection of bladder tumors are essential. For low-risk NMIBCs, BCG is not recommended, and, instead, patients should receive a single perioperative instillation of intravesical chemotherapy such as Gemcitabine, which has shown effectiveness, a better safety profile and lower cost compared to mitomycin, and is now the preferred agent for low-risk disease in 2019 National Comprehensive Cancer Network (NCCN) guidelines. ${ }^{9,10}$ For intermediate-risk NMIBC with low grade recurrent/multifocal disease, intravesical chemotherapy with gemcitabine, docetaxel or mitomycin, instead of BCG, are reasonable options. For high-risk NMIBC, all efforts should be made to treat with full-dose BCG, which has been proven superior to chemotherapy. ${ }^{11}$ In the setting of supply disruption, alternative treatment strategies should be employed to maximize the impact of the limited resource. One of the alternatives to be considered is reducing the dose to $1 / 3$ or a reduction of maintenance therapy from 3 years to 1 year for intermediate or high-risk patients without carcinoma in situ (CIS). In a study by Oddens et al, reducing the maintenance therapy from 3 years to 1 year was not inferior in intermediate-risk patients. For high-risk patients, there was an increase in cancer recurrence but no differences in mortality or progression. ${ }^{12}$ Other alternatives include induction BCG alone, reserving BCG maintenance therapy for patients with the highest risk disease. When BCG is not available, intravesical chemotherapy with mitomycin, gemcitabine, doxorubicin, docetaxel or sequential combination therapies might be offered. ${ }^{13}$ Finally, in patients with the highest risk of
Address for correspondence Alejandro Abello, MD,

Department of Urology, Yale School of Medicine/Yale-New Haven Hospital, 789 Howard Av, FMP 300. New Haven, CT, 06519, United States (e-mail: Alejandro. abello@yale.edu; Patrick.a. kenney@yale.edu).
DOI https://doi.org/ 10.1055/s-0039-1692693. ISSN 0120-789X. eISSN 2027-0119.
Copyright ( 2 2019, Sociedad Colombiana License terms de Urología. Publicado por Thieme Revinter Publicações Ltda., Rio de Janeiro, Brazil. Todos los derechos reservados. 
progression (e.g., T1 with CIS), a timely cystectomy should be considered. ${ }^{14}$

Moving forward, it is essential to identify new agents and improve our shared intravesical therapy supply chain. Trials that could improve reliability in treatment should be supported. The Southwest Oncology Group (SWOG) is conducting the SWOG 1602 trial, which is currently enrolling and randomizes patients with high-risk NMIBC to induction and maintenance with TICE strain vs. Tokyo strain vs. priming plus Tokyo strain. ${ }^{15}$ The trial not only reduces the current demand for the TICE strain, but it may add another BCG strain to our armamentarium, if favorable.

Supply disruptions are not unique to bladder cancer or BCG. In recent years, shortages of drugs and devices have resulted from natural disasters, manufacturing problems, market forces, and unintended consequences of regulatory intervention. ${ }^{16,17}$ These disruptions bring in to stark relief the essential work that must take place to ensure a more robust supply chain-including prioritization of a flexible supply base and avoidance of sole source suppliers, when feasible, along with proper incentives for manufacturers to improve reliability. ${ }^{18}$

\section{Conflict of Interest}

The authors declare that there is no conflict of interest.

\section{References}

1 Morales A, Eidinger D, Bruce AW. Intracavitary Bacillus CalmetteGuerin in the treatment of superficial bladder tumors. J Urol 1976;116(02):180-183

2 Sylvester RJ, van der MEIJDEN AP, Lamm DL. Intravesical bacillus Calmette-Guerin reduces the risk of progression in patients with superficial bladder cancer: a meta-analysis of the published results of randomized clinical trials. J Urol 2002;168(05): 1964-1970

3 Siegel RL, Miller KD, Jemal A. Cancer statistics, 2019. CA Cancer J Clin 2019;69(01):7-34

4 Bray F, Ferlay J, Soerjomataram I, Siegel RL, Torre LA, Jemal A. Global cancer statistics 2018: GLOBOCAN estimates of incidence and mortality worldwide for 36 cancers in 185 countries. CA Cancer J Clin 2018;68(06):394-424
5 Khanna A, Yerram N, Zhu H, Kim S, Abouassaly R. Utilization of Bacillus Calmette-Guerin for Nonmuscle Invasive Bladder Cancer in an Era of Bacillus Calmette-Guerin Supply Shortages. Urology 2019;124:120-126

6 Messing EM. The BCG Shortage. Bladder Cancer 2017;3(03): 227-228

7 Mostafid AH, Palou Redorta J, Sylvester R, Witjes JA. Therapeutic options in high-risk non-muscle-invasive bladder cancer during the current worldwide shortage of bacille Calmette-Guérin. Eur Urol 2015;67(03):359-360

8 NOTIFICATION OF SUPPLY ALLOCATION: TICE® BCG BCG LIVE (for intravesical use) [press release]. January, 20192019

9 National Comprehensive Cancer Network. NCCN Clincial Practice Guidelines in Oncology. Bladder Cancer 2018

10 Messing EM, Tangen CM, Lerner SP, et al. Effect of Intravesical Instillation of Gemcitabine vs Saline Immediately Following Resection of Suspected Low-Grade Non-Muscle-Invasive Bladder Cancer on Tumor Recurrence: SWOG S0337 Randomized Clinical Trial. JAMA 2018;319(18):1880-1888

11 Han RF, Pan JG. Can intravesical bacillus Calmette-Guérin reduce recurrence in patients with superficial bladder cancer? A metaanalysis of randomized trials. Urology 2006;67(06):1216-1223

12 Oddens J, Brausi M, Sylvester R, et al. Final results of an EORTC-GU cancers group randomized study of maintenance bacillus CalmetteGuérin in intermediate- and high-risk Ta, T1 papillary carcinoma of the urinary bladder: one-third dose versus full dose and 1 year versus 3 years of maintenance. Eur Urol 2013;63(03):462-472

13 Lightfoot AJ, Breyer BN, Rosevear HM, Erickson BA, Konety BR, O'Donnell MA. Multi-institutional analysis of sequential intravesical gemcitabine and mitomycin $C$ chemotherapy for non-muscle invasive bladder cancer. Urol Oncol 2014;32(01):35 e15-39

14 Important Message About the BCG Shortage [press release]. auanet.org, February 19, 2019

15 Svatek RS, Tangen C, Delacroix S, Lowrance W, Lerner SP. Background and Update for S1602 "A Phase III Randomized Trial to Evaluate the Influence of BCG Strain Differences and T Cell Priming with Intradermal BCG Before Intravesical Therapy for BCG-naïve High-grade Non-muscle-invasive Bladder Cancer. Eur Urol Focus 2018;4(04):522-524

16 Food and Drug Administration. Identifying the Root Causes of Drug Shortages and Finding Enduring Solutions; Public Meeting; Request for Comments 83 FR 45640. (2018-19612):45640-45642

17 Food and Drug Administration. Latest News on Sterigenics and Other Sterilization Facility Closures. 2019

18 Tang CS. Robust strategies for mitigating supply chain disruptions. International Journal of Logistics Research and Applications. 2006;9(01):33-45 